\title{
A NOTE ON EXTENSIONS OF BAER AND P. P. -RINGS
}

\author{
EFRAIM P. ARMENDARIZ
}

(Received 2 July 1973)

Communicated by $\mathbf{B}$. Mond

Baer rings are rings in which the left (right) annihilator of each subset is generated by an idempotent [6]. Closely related to Baer rings are left P.P.-rings; these are rings in which each principal left ideal is projective, or equivalently, rings in which the left annihilator of each element is generated by an idempotent. Both Baer and P.P.-rings have been extensively studied (e.g. [2], [1], [3], [7]) and it is known that both of these properties are not stable relative to the formation of polynomial rings [5]. However we will show that if a ring $R$ has no nonzero nilpotent elements then $R[X]$ is a Baer or P.P.-ring if and only if $R$ is a Baer or P.P.-ring. This generalizes a result of S. Jøndrup [5] who proved stability for commutative P.P.-rings via localizations - a technique which is, of course, not available to us. We also consider the converse to the well-known result that the center of a Baer ring is a Baer ring [6] and show that if $R$ has no nonzero nilpotent elements, satisfies a polynomial identity and has a Baer ring as center, then $R$ must be a Baer ring. We include examples to illustrate that all the hypotheses are needed.

We will assume throughout that rings have a unit. For convenience we call a ring reduced if it has no nonzero nilpotent elements. In a reduced ring $R$ left and right annihilators coincide for any subset $U$ of $R$, hence we let $\operatorname{ann}_{R}(U)=l(U)$ $=r(U)=\{a \in R \mid a U=0\}$.

The key lemma is the following characterization of zero divisors in $R[X]$ when $R$ is a reduced ring.

LEMMA 1. Let $R$ be a reduced ring and $f, g \in R[X]$ with $f=\sum_{i=0}^{n} a_{i} X^{i}$, $g=\sum_{i=0}^{m} b_{i} X^{i}$. Then $f g=0$ if and only if $a_{i} b_{j}=0$ for all $0 \leqq i \leqq n$, $0 \leqq j \leqq m$.

PROOF. Suppose that $f g=0$; we can assume that $n=m$. We then have equations: $a_{0} b_{0}=0 ; a_{1} b_{0}+a_{0} b_{1}=0 ; \cdots ; a_{n} b_{0}+\cdots+a_{0} b_{n}=0$. Since $R$ is reduced $a_{0} b_{0}=0$ if and only if $b_{0} a_{0}=0$. Hence left multiplying the second of these equations by $b_{0}$ yields $b_{0} a_{1} b_{0}=0$ hence $\left(a_{1} b_{0}\right)^{2}=0$ and so $a_{1} b_{0}=0$. Similarly we get $a_{i} b_{0}=0$ for all $1 \leqq i \leqq n$. Thus the original equations reduce to: $a_{0} b_{1}=0$; 
$a_{1} b_{1}+a_{0} b_{2}=0 ; \cdots ; a_{n-1} b_{1}+\cdots+a_{0} b_{n}=0$. Again using the fact that $a_{0} b_{1}=0$ implies $b_{1} a_{0}=0$ we conclude from the second equation that $a_{1} b_{1}=0$ and then similarly that $a_{i} b_{1}=0$ for all $1 \leqq i \leqq n$. Repetition yields $a_{i} b_{j}=0$ for all $1 \leqq i \leqq n, 1 \leqq j \leqq n$ as desired. The converse is clear.

As consequences we have

COROLLARY 1. If $R$ is a reduced ring and $f \in R[X]$ such that $f^{2}=f$ then $f \in R$.

Proof. Let $f=\sum_{i=0}^{n} a_{i} X^{i}$ so that $1-f=\left(1-a_{0}\right)+\sum_{i=1}^{n} a_{i} X^{i}$. From $f^{2}=f$ we get $a_{0}\left(1-a_{0}\right)=0$ and $a_{i}^{2}=0$ for all $i \geqq 1$. Hence $a_{i}=0$ for all $i \geqq 1$ and $f=a_{0}=a_{0}^{2} \in R$.

If $f \in R[X]$ of degree $n$ and $f=\sum_{i=0}^{n} a_{i} X^{i}$ let $S_{f}=\left\{a_{0}, a_{1}, \cdots, a_{n}\right\}$.

COROLlaRY 2. Let $R$ be a reduced ring and $U \subseteq R[X]$. If $T=U_{f=U} S_{f}$ then $\operatorname{ann}_{R[X]}(U)=\operatorname{ann}_{R}(T)[X]$.

ProOF. If $g=\sum_{i=0}^{m} b_{i} X^{i} \in R[X]$ and $g U=0$ then $g f=0$ for all $f \in U$ and hence for $0 \leqq i \leqq m, b_{i} \in \operatorname{ann}_{R}(T)$ by Lemma 1 . The opposite inclusion is clear.

THEOREM A. Let $R$ be a reduced ring. Then $R[X]$ is a P.P.ring if and only if $R$ is a P.P.ring.

Proof. If $R[X]$ is a P.P.-ring and $a \in R$ then $\operatorname{ann}_{R}(a)=R \cap \operatorname{ann}_{R[X]}(a)$ $=R \cap R[X] e$ with $e^{2}=e$. By Corollary $1, e \in R$ and thus $R \cap R[X] e=R e$. Now assume $R$ is a P.P.-ring. First note that if $a, b \in R$ with $\operatorname{ann}_{R}(a)=R e_{1}$, $\operatorname{ann}_{R}(b)=R e_{2}$ where $e_{1}^{2}=e_{1}, e_{2}^{2}=e_{2}$ then if $e=e_{1}+e_{2}-e_{1} e_{2}$ we have $e^{2}=e$ because idempotents of $R$ are central and $\operatorname{ann}_{R}(\{a, b\})=R e$. Thus for any finite subset $U \subseteq R, \operatorname{ann}_{R}(U)=R e$ for some idempotent $e \in R$. If $f \in R[X]$ then by Corollary 2, $\operatorname{ann}_{R[X]}(f)=\operatorname{ann}_{R}\left(S_{f}\right)[X]=R e[X]=R[X] e$ with $e^{2}=e$, as $S_{f}$ is finite. Thus $R[X]$ is a P.P.-ring.

Similarly one can establish

THEOREM B. Let $R$ be a reduced ring. Then $R[X]$ is a Baer ring if and only if $R$ is a Baer ring.

Let $R$ be the ring of all $2 \times 2$ matrices over the integers. Then $R$ is a Baer ring [6] but $R[X]$ is not Baer since it is not P.P. because the left annihilator of $\left[\begin{array}{ll}2 & 0 \\ x & 0\end{array}\right]$ is not generated by an idempotent. This example is given in [5] and is due to P. M. Cohn. Note also that the center of $R[X]$ is a Baer ring thus a ring with center a Baer ring need not be a Baer ring. This example is not a reduced ring, thus one might conjecture that if $R$ is reduced and its center is a Baer ring then $R$ is a Baer ring. However this is not the case and here is a counterexample. Let $K$ be a field, $X, Y, Z$ indeterminates and take $R=K[X, Y, Z]$ subject to $X Y=X Z$ $=Z X=Y X=0$ and $Y Z \neq Z Y$. Then $R$ is a reduced ring with center $K[X]$ and $K[X]$ is a Baer ring. Also ann $(Y)=R X=K[X] X$ and so $\operatorname{ann}(Y)$ contains no 
nonzero idempotents. If we assume in addition that $R$ satisfies a polynomial identity then the conjecture is true. In order to establish this we record a result on reduced rings with a polynomial identity which is of independent interest. We recall that a ring $R$ satisfies a polynomial identity if there is a polynomial $p\left(X_{1}, \cdots, X_{n}\right)$ in non-commuting indeterminates with coefficients from the centroid of $R$ such that $p\left(a_{1}, \cdots, a_{n}\right)=0$ for all choices $a_{1}, \cdots, a_{n}$ of elements from $R$. Since we are dealing with reduced rings with 1 , the coefficients come from the center of $R$; see [4].

THEOREM C. Let $R$ be a reduced ring satisfying a polynomial identity. Then each nonzero left (right) ideal of $R$ contains a nonzero two-sided ideal of $R$.

Proof. If $I$ is a nonzero left ideal of $R$ choose $0 \neq a \in I$ so that $R a$ satisfies a multilinear identity $p\left(X_{1}, \cdots, X_{k}\right)$ of minimal degree among left ideals $R b \subseteq I$ with $b \neq 0$. Write

$$
p\left(X_{1}, \cdots, X_{k}\right)=p_{1}\left(X_{2}, \cdots, X_{k}\right) X_{1}+p_{2}\left(X_{1}, \cdots, X_{k}\right)
$$

where the monomials occurring in $p_{2}$ do not end with $X_{1}$. Since $a^{2} \neq 0$ there exist $u_{2} a^{2}, \cdots, u_{k} a^{2}$ in $R a^{2}$ so that $d=p_{1}\left(u_{2} a^{2}, \cdots, u_{k} a^{2}\right) \neq 0$. Letting $X_{1}=r a$ for any $r \in R$, we get $0=p\left(r a, u_{2} a^{2}, \cdots, u_{k} a^{2}\right)=d r a+s a^{2}$ for some $s \in R$ and so $d r+s a \in \operatorname{ann}(a)$. Hence $d R \subseteq R a+\operatorname{ann}(a)$ and thus $a d R \subseteq a R a \subseteq I$. Moreover $a d R \neq 0$ since $d \in R a^{2}$ and $R a^{2} \cap \operatorname{ann}\left(a^{2}\right)=0$. Thus $R(a d) R$ is a nonzero ideal of $R$ lying in $I$.

As a consequence of Theorem $C$, we have that if $I$ is a nonzero ideal of a reduced ring with a polynomial identity then summing all two-sided ideals contained in $I$ we get a largest two-sided ideal $I_{0} \subseteq I$, and $I_{0}$ is essential in $I$. Since the singular ideal of a reduced ring is zero [8], it follows that $\operatorname{ann}\left(I_{0}\right)=\operatorname{ann}(I)$.

THEOREM $D$. Let $R$ be a reduced ring satisfying a polynomial identity. If the center of $R$ is a Baer ring then $R$ is a Baer ring.

Proof. Let $A=$ center $R, \phi \neq T \subseteq R$ and $I=\sum_{t \in T} R t$. Then $\operatorname{ann}_{R}(T)$ $=\operatorname{ann}_{R}(I)=\operatorname{ann}_{R}\left(I_{0}\right)$. Let $B=I_{0} \cap A$; since $A$ is Baer, $\operatorname{ann}_{A}(B)=A e$ for some idempotent $e \in A$. We wish to show that $R e=\operatorname{ann}_{R}(T)$. If $e t \neq 0$ for some $t \in T$, then Ret $\subseteq I$. Hence if $0 \neq W$ is an ideal of $R$ contained in Ret then $W \subseteq I_{0}$. By a theorem of Rowen [9], $W \cap A \neq 0$ so if $0 \neq w \in W \cap A_{0}$ then $w=x e t$ and $w \in B$ implies $w^{2}=0$ so $w=0$ a contradiction. It follows that $R e \subseteq \operatorname{ann}_{R}(T)$. If $0 \neq y \in \operatorname{ann}_{R}(T)$ then $R y$ contains a nonzero ideal $U$ of $R$ so again by [9], $U \cap A \neq 0$. Thus

$$
0 \neq x y \in A \cap \operatorname{ann}_{R}(T)=\operatorname{ann}_{A}(B)
$$

so $x y=x y e$. Thus $R e$ is essential in $\operatorname{ann}_{R}(T)$. Since $\operatorname{ann}_{R}(T)=\operatorname{Re} \oplus\left[\operatorname{ann}_{R}(T)\right.$ $\cap R(1-e)]$ it follows that $R e=\operatorname{ann}_{R}(T)$ as desired. 


\section{References}

[1] G. Bergman, 'Hereditary commutative rings and centres of hereditary rings', Proc. London Math. Soc. 23 (1971), 214-236.

[2] S. Endo, 'A Note on pp. rings', Nagoya Math. J. 17 (1960), 167-170.

[3] M. Evans, 'On commutative P. P. rings', Pacific J. Math. 41 (1972), 687-697.

[4] N. Jacobson, Structure of Rings, (Amer. Math. Soc. Colloq. Publ. v. 37, Providence, R. I. (1964).)

[5] S. Jøndrup, 'p. p. Rings and finitely generated flat ideals', Proc. Amer. Math. Soc. 28 (1971), 431-435.

[6] 1. Kaplansky, Rings of Operators, (W. A. Benjamin, New York (1968)).

[7] T. Speed, 'A note on commutative Baer rings', J. Austral. Math. Soc. 14 (1972), 257-263.

[8] G. Renault, 'Anneaux reduits non commutatifs', J. Math. Pures et Appl. 46 (1967), 203-214.

[9] L. Rowen, 'Some results on the center of a ring with polynomial identity', Bull. Amer. Math. Soc. 79 (1973), 219-223.

University of Texas

Austin, Texas 78127

U. S. A. 